\title{
La bataille de l'autisme Réflexions sur un phénomène social contemporain
}

\author{
J. Hochmann ${ }^{1}$ \\ (C) Springer-Verlag 2009
}

\section{Introduction}

L'autisme infantile précoce a été décrit, en 1943, par le pédopsychiatre américain Leo Kanner autour de deux symptômes cardinaux : l'isolement (aloneness) et le besoin d'immuabilité (sameness) [23]. Dans les nomenclatures modernes (DSM IV, CIM10 et CFTMEA), il est caractérisé, on le sait, par l'association :

- de troubles de la communication (un dysfonctionnement précoce de la communication non verbale et, ensuite, une absence de langage articulé ou des modifications dans sa syntaxe et son énonciation) ;

- des troubles de la socialisation (un retrait sur soi et une indifférence apparente à autrui, un défaut de recours à l'autre dans les situations de détresse, une difficulté dans le jeu symbolique et le faire semblant) ;

- et d'intérêts restreints (stéréotypies gestuelles, rituels, résistance au changement, fixation sur des formes ou des objets particuliers dits autistiques).

Ces troubles doivent être apparus avant l'âge de trois ans. Ils s'accompagnent le plus souvent d'un retard mental mais peuvent coexister avec une intelligence normale, voire supérieure dans certains domaines, à l'origine de talents dits paradoxaux. Lorsque le besoin d'immuabilité est contré, nombre d'enfants autistes montrent des signes d'angoisse majeure et peuvent présenter des troubles du comportement dont le plus dramatique est l'automutilation. Longtemps tenu pour une rareté, l'autisme a vu sa prévalence s'accroître de manière exponentielle. On ne peut manquer de s'interroger sur cette véritable épidémie autistique ainsi que sur la mode de l'autisme qui envahit la presse, la télévision et l'édition. L'autisme infantile est, en effet, devenu depuis peu un

\section{J. Hochmann $(\bowtie)$}

Centre hospitalier le Vinatier, 95, boulevard Pinel,

F-69677 Bron, France

e-mail : hochmann.jacques@wanadoo.fr

\footnotetext{
${ }^{1}$ Professeur émérite de psychiatrie de l'enfant à l'université ClaudeBernard. Médecin honoraire des hôpitaux de Lyon. Auteur de Histoire de l'autisme, Odile Jacob, Paris, 2009.
}

phénomène social, et le mot lui-même connaît une véritable glissade sémantique. Tous les jours, dans les meilleurs journaux et jusqu'à l'Assemblée nationale, on taxe d'autiste un dirigeant parce qu'il ne tient pas compte de ses subordonnés ou un homme politique parce qu'il est insensible aux signaux de ses électeurs. L'origine ultime de l'autisme reste inconnue, et cette énigme explique en partie les attitudes passionnelles de ceux qui, pour la résoudre, se sont dressés les uns contre les autres, au fil des années, et imposent aujourd'hui à la pensée un totalitarisme, voire une censure dont on trouve peu d'autres exemples dans les débats scientifiques des pays démocratiques.

La véritable bataille de l'autisme qui, en dépit d'accalmies temporaires et d'armistices de circonstances, continue à faire rage sur Internet et dans les médias, porte essentiellement sur trois points :

- les limites de l'autisme et sa place dans la nosographie : psychose ou trouble envahissant du développement, maladie ou handicap ;

- la nature et l'origine de ce trouble : déficit ou défense, trouble inné ou acquis de nature neurologique, ou plutôt réaction psychologique aux facteurs d'environnement, notamment maternels ;

- le type de prise en charge la plus indiquée: soin relationnel à visée psychothérapique ou éducation unilatérale centrée sur la modification du comportement.

On essaiera ici de décrire les trois facettes de ce curieux phénomène social, sans doute exacerbé par la souffrance qu'éprouvent les familles d'enfants autistes, mais dont on peut trouver des racines dans l'histoire. En effet, avant d'être décrits sous le nom d'autistes, ces enfants confondus avec d'autres arriérés mentaux et appelés idiots faisaient l'objet de disputes analogues entre les tenants de l'hérédité et ceux d'une carence environnementale, entre les éducateurs et les médecins. Le terme d'idiot connaissait alors une dérive identique à celle qui affecte aujourd'hui la dénomination d'autiste et des eugénistes, notamment aux États-Unis, redoutaient une prolifération semblable à celle que revendiquent aujourd'hui certaines associations de parents, sur la foi d'enquêtes épidémiologiques [21]. Le 
phénomène nouveau est le pouvoir de ces associations dans le cadre d'une société consumériste où l'usager a fort heureusement acquis de nouveaux droits, mais où les techniques modernes de communication laissent souvent le dernier mot à celui qui crie le plus fort et qui a le plus de moyens pour se faire entendre. Certains de ces moyens sont économiques, d'autres idéologiques ou affectifs, comme l'usage compassionnel d'une détresse, à une époque où le statut de victime fait recette.

\section{Limites de l'autisme}

Kanner, dès 1943, considérait l'autisme comme une maladie rare et, évaluant sa prévalence à environ un cas pour dix mille, il s'élevait contre un laxisme qui, selon lui, aboutissait à multiplier abusivement les diagnostics. "Presque en une nuit, ironisait-il dans un de ses articles, le pays parut peuplé par une multitude d'enfants autistes ». Que dirait-il aujourd'hui ? Après que les spécialistes aient parlé de cinq cas pour dix mille, puis d'un cas pour mille, l'association des familles américaines d'autistes, annonce, avec l'existence d'un " spectre autistique », un cas pour 150, un taux qui a doublé en un an dans une enquête californienne et qui augmente chaque année de $15 \%$, laissant prévoir, en 2010, quatre millions d'autistes pour les seuls États-Unis, soit plus de $1 \%$ de la population.

Pour expliquer cette étrange prolifération plusieurs hypothèses ont été avancées :

- on passera sur des hypothèses dont de nombreuses études ont démontré le caractère fantaisiste : une intoxication par les métaux lourds, un virus ou encore la sensibilité à un composé organomercurique utilisé pour stabiliser le vaccin ROR. Cette dernière hypothèse entretient encore une campagne contre cette vaccination, ce qui explique la réapparition de rougeoles parfois mortelles, alors qu'on croyait cette maladie définitivement éradiquée ;

- l'hypothèse la plus vraisemblable est une extension du dépistage, aujourd'hui de plus en plus précoce. Cette évolution des pratiques est heureuse, car elle permet des interventions thérapeutiques ou/et éducatives avant que le trouble ne soit davantage fixé. Elle s'inscrit toutefois dans une extension du diagnostic qui mérite qu'on s'y arrête. Comme il n'existe aucun test biologique, aucune image cérébrale, aucune anomalie de tracé électroencéphalographique spécifique de l'autisme, le diagnostic ne peut reposer que sur des signes cliniques regroupés consensuellement en syndrome par les cliniciens. Or, c'est ici que le bât blesse. Kanner et ses premiers successeurs avaient défini un certain nombre de critères précis que nous avons rappelés et sur lesquels s'accordaient les spécialistes du monde entier. Ce sont encore ceux de l'autisme de Kanner dit «typique » repris dans les différentes classifications. Alors que les auteurs de l'époque s'accordaient généralement avec Kanner pour distinguer l'autisme de la schizophrénie infantile (un diagnostic devenu très vague qui recouvrait aux ÉtatsUnis une grande partie des troubles du comportement), le terme de psychose infantile avait prévalu. Il avait été proposé par Mahler pour distinguer un trouble grave de la relation à autrui et du contact avec la réalité distinct, chez un être encore en développement, du processus de dissociation d'une individualité constituée, caractéristique, depuis Bleuler, des schizophrénies de l'adulte. Se basant sur une théorie du développement normal, fondée sur des observations de bébés en crèche et de leurs relations avec un adulte nourricier, Mahler distinguait deux types de psychose: les psychoses autistiques, décrites par Kanner, où l'objet maternel n'est pas individualisé comme "balise d'orientation émotionnelle », et les psychoses symbiotiques, révélées plus tardivement, où l'enfant s'enferme dans l'illusion quasi délirante d'une «unité duelle » formée avec sa mère et ne parvient pas ainsi à s' « individuer» [26]. Cette distinction devait être reprise par les auteurs français, Roger Misès proposant le terme de "dysharmonie psychotique » pour regrouper les psychoses symbiotiques et certaines formes plus tardives contemporaines de la période de latence. Sur des critères psychopathologiques, il opposait ces dysharmonies psychotiques, structurées par des mécanismes de défense du type de l'identification projective et du clivage de l'objet, aux psychoses autistiques, où domine l'identification adhésive à un objet sans profondeur avec lequel le lien n'est que celui d'une sensorialité éclatée, attentive aux seuls effets de surface. Il proposait de distinguer une troisième catégorie, celle des psychoses déficitaires, où les éléments lésionnels peuvent être au premier plan, mais qui permettaient de replacer un certain nombre d'arriérations mentales dans le registre du soin et d'inspirer des pratiques plus optimistes et plus fécondes que le seul gardiennage assorti d'un conditionnement élémentaire, en lui-même débilisant. Il réservait le diagnostic de schizophrénie infantile à des formes rares de schizophrénie apparue avant ou aux alentours de la puberté et qui évoluaient ensuite vers une schizophrénie de l'adulte, ce qui n'était pas le cas des psychoses infantiles [30].

Tant le diagnostic d'autisme que celui de psychose avaient pour objectif d'arracher un nombre conséquent d'enfants à l'optique désespérante qui, depuis les travaux de Binet et Simon, à l'origine de la mesure de l'intelligence, prévalait dans le domaine de l'arriération mentale profonde. En dessous d'un certain QI (autour de 50), les enfants, en ce temps, étaient considérés comme des idiots congénitaux voués à la ségrégation et parfois à la castration ou à la 
stérilisation. Kanner, à tort, insistait sur l'intelligence normale de ses autistes, mais c'était pour leur éviter le sort des arriérés. Margaret Mahler puis, en Angleterre, les élèves de Klein et, en France, des psychanalystes d'enfants comme Serge Labovici, René Diatkine, Roger Misès ou Jean-Louis Lang se référaient à la notion de psychose (et à la psychopathologie d'inspiration psychanalytique) pour promouvoir des programmes thérapeutiques, éducatifs et pédagogiques là où, jusqu'alors, depuis un demi-siècle, ne régnait que l'abandon. La référence médicale à une maladie (qui était la référence de Kanner), donc à des soins, avait alors, très concrètement, l'avantage de permettre de bénéficier des enveloppes sanitaires, plus fournies que les enveloppes médicosociales davantage consacrées à l'accueil, à la rééducation et à la réhabilitation du handicap. Dans des asiles surencombrés, ces auteurs initièrent une politique de réaménagement et d'humanisation des locaux ainsi que d'équipement en personnel : des médecins et des psychologues psychanalystes mais aussi des infirmiers et infirmières, des éducateurs spécialisés, des orthophonistes, des psychomotriciens, des instituteurs détachés de l'Éducation nationale. En 1960, Serge Lebovici ouvrait à Paris le premier hôpital de jour, bientôt imité par d'autres dans la capitale et en province, à l'origine des secteurs de psychiatrie infantojuvénile dont les équipes développaient les premières tentatives d'intégration en milieu scolaire normal. Parallèlement, les associations de parents regroupées autour de professionnels, comme Robert Lafon à Montpellier, Claude Kohler à Lyon, Georges Heuyer à Paris, avaient construit toute une gamme d'institutions médicosociales, qui modifiaient en profondeur le paysage de l'enfance dite inadaptée. Un partage semblait s'être établi, les secteurs de psychiatrie infantojuvénile prenant en charge le vaste champ des troubles névrotiques et les soins précoces des psychoses et des organisations pathologiques dites « limite », le secteur médicosocial se réservant une large part de la déficience mentale et des troubles du comportement, ainsi que les suites à l'adolescence des troubles psychotiques de l'enfance. Si une certaine atmosphère de concurrence et, longtemps, une réglementation qui s'opposait aux doubles prises en charge ralentirent la recherche des complémentarités et les coopérations, cellesci tendirent ensuite à se multiplier avec souvent des recouvrements entre les deux types de structure, pendant qu'une nouvelle conception plus dynamique du handicap permettait de ne plus l'opposer à la maladie.

C'est alors que certaines associations de famille, d'abord aux États-Unis, puis dans tout le monde occidental, ignorant cette évolution conceptuelle, entrèrent en guerre contre la notion de psychose et contre celle de maladie qui lui était liée. Il y avait à cela plusieurs raisons. D'une part, depuis le début des années 1970, les familles d'autistes américains avaient commencé à s'opposer aux approches d'inspiration psychanalytique vécues, non sans raison, comme culpabilisantes et comme source d'espoirs non tenus. D'autre part, les restrictions des budgets fédéraux de la santé mentale, particulièrement drastiques avec l'avènement au pouvoir de Ronald Reagan en 1980, mais déjà esquissées avant, rendaient plus avantageux le recours à des institutions éducatives, au nom du droit de tout jeune américain à l'éducation, inscrit dans la constitution des États-Unis. Dès 1975, ces familles avaient obtenu l'inscription de l'autisme parmi les developmental disabilities ouvrant droit à compensation au même titre que l'épilepsie, que le retard mental et que l'infirmité motrice cérébrale. Sous leur pression, le principal journal scientifique sur le sujet, fondé par Kanner, le Journal of autism and childhood schizophrenia, était devenu, en 1979, le Journal of autism and developmental disorders. Le DSM III, la nouvelle édition de la classification américaine des troubles mentaux, ne fit qu'entériner ce changement de nom. Réservé aux troubles de l'adulte, le terme de psychose disparut du vocabulaire de la pédopsychiatrie et, avec lui, toute allusion à la psychopathologie et à la psychanalyse. Aucune recherche sérieuse n'avait pourtant montré l'inefficacité des traitements engagés jusque-là ni permis encore d'affirmer une étiologie organique, soupçonnée depuis longtemps mais toujours en attente de preuve. Le principal artisan de la manœuvre, le Dr Rimland, un psychologue de la marine américaine, spécialiste des tests et lui-même père d'autiste, fondateur de l'Autism Society of America, avait bien proposé une première théorie qui faisait intervenir la substance réticulée du tronc cérébral, dont Moruzzi et Magoun avait montré, dans les années 1960, le rôle dans l'activation du système nerveux central, mais cette hypothèse avait fait long feu, comme celle qu'il proposa ensuite d'une carence en vitamine B6 [33]. Il est important de le noter, contrairement à ce qui est souvent affirmé, « le grand renversement » (pour reprendre un terme d'Alain Ehrenberg), au moins dans le domaine de l'autisme, précéda les découvertes biologiques (dont on verra plus loin le caractère encore aujourd'hui modeste). Ce fut donc un renversement purement idéologique et non un changement de paradigme lié à une avancée expérimentale (evidence-based).

Il est intéressant de suivre ensuite l'évolution du concept nouveau au fil des éditions successives du DSM (DSM IIIR, DSM IV, DSM IVR). Au départ, l'autisme figure parmi les troubles globaux du développement qui s'opposent aux troubles spécifiques isolés, portant sur un secteur du comportement : l'apprentissage de la lecture, la parole, la conduite, l'activité motrice, l'attention, etc. Puis il devient un des troubles envahissants du développement (TED), aux côtés du syndrome de Rett (cette maladie dégénérative atteignant les petites filles, et dont on connaît aujourd'hui le gène responsable), les troubles désintégratifs de l'enfance (des atteintes neurologiques liées généralement à un 
processus encéphalopathique dégénératif) et l'entité des troubles envahissants du développement non spécifiés autrement. Le syndrome de Rett et les troubles désintégratifs étant très rares, ne restent pratiquement face-à-face que l'autisme et les troubles non spécifiés autrement, vaste et confuse catégorie sans signes positifs propres.

C'est alors que se produisent trois phénomènes concomitants. Par ailleurs, sous l'influence d'une pédopsychiatre anglaise, Lorna Wing, on redécouvre un auteur autrichien complètement oublié qui, en même temps que Kanner, avait utilisé le même vocable pour décrire un syndrome très voisin sinon identique qu'il avait appelé "psychopathie autistique »[1]. On décide de donner le nom de syndrome d'Asperger à des cas d'autisme léger sans atteinte de l'intelligence, avec un langage d'apparition précoce, parfois précieux dans le vocabulaire mais d'une syntaxe normale, une fréquente maladresse motrice et un trouble dominant des relations sociales. La définition très imprécise de ce syndrome (que certains identifient à l'autisme dit de « haut niveau » mais dont d'autres font simplement une forme particulière d'intelligence voisine de la normalité) explique le nombre croissant à l'infini de sujets qualifiés Asperger. Ce diagnostic permet, aux États-Unis et au Canada, à des élèves mal adaptés au système scolaire, d'obtenir, avec la reconnaissance de leur état, une scolarité aménagée et éventuellement une aide financière, aménagement et aide qu'un jeune français peut, à la même époque, obtenir de la Commission départementale de l'éducation spécialisée sur simple évaluation de ses difficultés et sans diagnostic particulier. À l'autre bout, on assouplit les critères de diagnostic de l'autisme atypique, par son apparition plus tardive, son tableau clinique incomplet. Surtout, sous la pression de familles, qui veulent bénéficier de la même reconnaissance que celle obtenue par les parents d'autistes, on élargit le diagnostic d'autisme syndromique (c'est-à-dire de traits autistiques associés à une affection génétique connue telle que le syndrome du $\mathrm{X}$ fragile, la sclérose tubéreuse de Bourneville, le syndrome d'Angelman ou celui de Prader-Willi) à des arriérations mentales et à des encéphalopathies de toutes sortes. À l'origine un des troubles envahissants du développement, l'autisme forme, dans la dernière édition du DSM, le noyau de ces troubles, les autres se distinguant de lui par leur plus ou moins grand degré de ressemblance avec la figure centrale. La notion de trouble du spectre autistique (TSA) tend alors à se substituer à celle de trouble envahissant du développement.

De plus, les modalités techniques les plus répandues du diagnostic peuvent aboutir à une multiplication des cas d'autisme. À défaut d'examens de laboratoire ou d'imagerie, qui jusqu'à présent ne révèlent, au mieux, que des troubles associés et ne peuvent, en aucun cas, permettre de dire qu'un enfant est autiste ou non, le diagnostic n'est que clinique. Or, la clinique, lorsqu'il s'agit d'un trouble affectant le fonctionnement global de la personne, son comportement, sa manière de gérer ses émotions, sa manière de penser, repose sur deux approches possibles. L'une ressort de la perception : avec ses yeux, en regardant agir un individu, avec ses oreilles, en l'écoutant parler, on peut repérer un certain nombre de symptômes. Ce sont ceux sur lesquels il est le plus facile de s'accorder. Dans un souci de fiabilité et de fidélité interjuge ce sont ceux qu'a retenus le DSM. De manière à standardiser les observations, il s'appuie sur des échelles qui permettent de chiffrer l'enregistrement audiovisuel des comportements allant du plus normal au plus anormal. Les trois plus connues sont l'ADI (Autistic Diagnostic Interview), l'ADOS (Autistic Diagnostic Observation Schedule) et la CARS (Childhood Autism Rating Scale) qui est plus une échelle de gravité que de diagnostic. L'ADI utilise une observation indirecte. C'est un entretien structuré des parents avec un professionnel qui les interroge sur l'évolution de leur enfant et retrace, avec leur aide, l'émergence de leurs principales inquiétudes. Il est souvent vécu par les parents comme un moment de réflexion et de prise de conscience qui leur donne le sentiment d'être enfin entendus et pris en compte. Mais il ne met pas à l'abri de biais. À une époque où les parents sont dûment informés sur l'autisme et ses symptômes par Internet et les médias, ils peuvent tendre à répondre dans un sens prédéterminé et être involontairement à l'origine de faux-positifs. Leurs réponses à l'ADI peuvent en effet être influencées par le désir sous-jacent de voir reconnaître à leur enfant un handicap inscrit dans la loi, qui permet, en occultant sa pathologie, de jeter un voile sur sa souffrance et leur évite aussi de vaines illusions. À tort ou à raison, ils pensent que ce handicap ouvre des portes dans des institutions spécialisées et permet de bénéficier de méthodes adéquates. Ils veulent aussi obtenir un diagnostic précis dont ils attendent une identité socialement plus valorisée que celle de malade mental ou d'arriéré et moins vague que celle de «traits autistiques» ou, a fortiori, de troubles envahissants du développement non spécifiés autrement. La formation à la pratique de l'ADI ne tient généralement pas compte de ces éléments «transférentiels », au sens large, et accepte, sans critique, comme vérité objective, les réponses des parents. L'ADOS et la CARS sont basées sur des observations, standardisées pour la première, en milieu naturel pour la seconde, faites par des professionnels formés mais qui ne sont pas incités à aller au-delà de ce qui se voit et s'entend en surface.

Un diagnostic plus précis pourrait reposer sur un autre type d'informations. Qu'on l'appelle «intuition» à la manière de Bergson ou «empathie » à l'instar des psychologues allemands de la fin du XIX ${ }^{e}$ siècle et des phénoménologues, il existe, à côté de la perception, une autre manière de percevoir qui permet (avec un certain entraînement) d'entrer dans l'intimité d'un sujet, de 
comprendre le sens de ce qu'il éprouve, de partager, jusqu'à un certain point, ses états mentaux, de résonner à ses types d'angoisse. L'empathie fait aujourd'hui l'objet de travaux scientifiques qui se fondent sur la découverte dans le cerveau des systèmes miroirs qui sont activés par les représentations d'action et les émotions d'autrui [35]. On sait que ces systèmes, miroirs indispensables à la connaissance d'autrui, sont en relation avec des circuits complexes générateurs de représentations partagées à la base du sentiment d'appartenance commune à une même culture, à une même condition [16]. L'empathie (ou l'intuition) a toujours fait partie de la démarche clinique (au moins jusqu'au DSM III). Un psychiatre hollandais des années 1930, Rümke, faisait ainsi, sans scandaliser personne, du praecox gewoel, du « sentiment précoce », un des éléments essentiels du diagnostic de schizophrénie. L'empathie est à la base des processus d'identification et de ce qu'en psychanalyse, on appelle le jeu transféro-contretransférentiel. Grâce à ce mécanisme, on peut dépasser le simple codage des comportements et s'interroger sur leur signification pour l'individu. C'est par l'empathie, telle qu'on l'exerce dans la relation qu'on noue avec lui, qu'on peut ressentir la manière qu'a l'enfant d'entrer en relation avec les objets animés et inanimés, qu'on peut distinguer, au moins de manière hypothétique, s'il contracte avec un objet total ou avec un objet partiel, avec un objet bidimensionnel ou avec un objet doté de profondeur, s'il prête à cet objet des intentions persécutoires ou s'il peut apprécier son caractère secourable, etc. Une brillante clinicienne américaine des années 1950, Bender, faisait ainsi état des sensations d'écoulement qu'elle éprouvait en prenant un enfant psychotique de type symbiotique dans ses bras [4]. Ces distinctions, passées sous silence, quand on se contente d'une observation rapportée par un tiers ou du remplissage d'un questionnaire, aussi détaillé soit-il, amènent à supposer, chez les enfants souffrant de troubles graves du développement, derrière une même façade apparemment autistique, des modalités relationnelles variées, justiciables d'approches pychothérapiques et éducatives différentes. Une telle approche peut être considérée comme subjective, largement conjecturale et non scientifique, mais, dans un domaine où il s'agit de diagnostiquer un trouble de l'intersubjectivité, cette « théorie de l'esprit » est peut-être épistémologiquement plus solide qu'une objectivité illusoire, fondée sur des manuels et des arbres de décision précontraints qui fabriquent artificiellement du consensus. Le fait est qu'elle réduit considérablement la population d'autistes, mais elle peut contribuer à affiner et à individualiser les indications de traitements. Les premiers psychopathologues ont décomposé l'idiotie puis la schizophrénie infantile. Il est temps peut-être de décomposer l'autisme qui est à son tour devenu ce que Kanner appelait un hodgepodge, un salmigondis de troubles divers, confondus dans une masse informe, un analogue de ce qu'étaient les fièvres dans les nosographies du XVIII siècle. La classe des enfants et des familles ayant besoin d'aides spécifiques est probablement nombreuse, mais il n'y a aucun avantage ni pour la recherche, ni pour l'amélioration des pratiques éducatives ou thérapeutiques à prendre un des éléments de cette classe pour la classe entière.

\section{Question de l'origine}

Il n'y a aucun avantage non plus à lui attribuer une origine unique. Nul ne peut honnêtement, aujourd'hui pas plus qu'hier, définir la cause de l'autisme.

Comme l'inconnu est toujours angoissant, on fait comme si on savait, et on multiplie encore, comme on a multiplié jadis, les affirmations dogmatiques. La tactique habituelle, inspirée du marketing, consiste à schématiser et à caricaturer l'opinion qu'on veut remplacer, pour mieux en démontrer le caractère archaïque et définitivement dépassé.

\section{Hypothèses psychanalytiques}

La tarte à la crème des journaux scientifiques, aussi bien que des médias généralistes, consiste ainsi à affirmer que, pendant un demi-siècle, les psychanalystes ont mis en cause l'influence supposée délétère de la mère et qu'avec l'abandon de cette théorie nocive la psychanalyse n'a plus rien à dire sur l'autisme. Qu'en est-il en réalité ?

Les premiers à avoir décrit un portrait type des parents d'autistes n'étaient pas des psychanalystes. Kanner, comme Asperger, l'un pédopsychiatre d'inspiration psychobiologique, l'autre pédiatre, avaient été frappés par le caractère froid et distant des pères, généralement des intellectuels plus préoccupés par de hautes spéculations ou par leur carrière que par leurs relations avec leurs enfants. Les mères, selon eux, ne s'intéressaient que superficiellement à leur progéniture et méritaient parfois l'étiquette de "mère frigidaire » forgée par Kanner (et non, comme on l'écrit souvent, par Bettelheim). Il est vrai que Kanner devait ensuite revenir partiellement sur ses premières constatations. Il remarquait que les autres enfants, élevés pourtant dans le même milieu, n'étaient pas autistes, qu'on trouvait parfois des enfants autistes chez des parents d'allure différente et que certains parents identiques à ceux qu'il avait décrits n'avaient pas d'enfant autiste. Il parlait d'un trouble inné du contact affectif, analogue à un trouble sensoriel ou moteur congénital. Plus tard, il devait s'indigner contre la tendance de plus en plus répandue à « chercher la mère » (en français dans le texte) et se résoudre à "acquitter » les mères de toute responsabilité, une responsabilité qu'il avait pourtant contribué à établir. Il concluait que l'autisme était quelque chose de trop 
compliqué pour être le simple produit d'un environnement, et que les traits de caractère parentaux étaient une constatation clinique dont il était difficile de dire si elle était la cause ou la conséquence de l'autisme de l'enfant [5]. Asperger tenait un discours voisin et y ajoutait l'hypothèse génétique de traits autistiques a minima transmis à l'enfant par les parents et aggravés chez lui. Cette hypothèse était dérivée de la théorie de la dégénérescence, qui avait dominé le siècle précédent. Elle postulait la transmission progressive dans une famille d'une tare héréditaire d'abord extériorisée par de simples stigmates physiques (un strabisme, un doigt surnuméraire, une déformation de la physionomie, du nez ou des oreilles) ou psychiques (une tendance obsessionnelle, une appétence aux toxiques ou à l'alcool, une prodigalité excessive ou une orientation sexuelle qualifiée de perverse). De génération en génération, cette tare s'aggravait pour produire tout le catalogue des troubles mentaux et pour aboutir, en fin de course, à l'idiotie, terme ultime de ce processus fatal qui a notamment inspiré Les Rougon Macquart d'Émile Zola.

Freud, on le sait, s'est élevé contre le caractère pessimiste et manifestement inexact de cette théorie, souvent infiltrée de jugements moraux, qui mettait dans le même sac toute une série de troubles sans rapport les uns avec les autres [14]. Sa première conceptualisation, qui attribuait les névroses à un traumatisme sexuel vécu dans l'enfance et retrouvant sens après coup au moment de l'éclosion de la puberté, était construite comme un contre-type de la théorie de la dégénérescence. Elle visait à donner au médecin une possibilité d'intervenir. On ne peut rien sur une tare transmise de manière inexorable (sinon éviter les croisements néfastes et la reproduction des tarés en les isolant ou en les stérilisant). On peut, par contre, aider le sujet à prendre conscience d'un traumatisme dont la représentation refoulée est devenue inconsciente, et les affects liés à cette représentation continuent à le faire souffrir. On sait aussi que Freud, dans son élaboration ultérieure, devait relativiser le rôle accordé au début au traumatisme réellement advenu et laisser toute la place au fantasme, c'est-à-dire à l'activité psychique du sujet, à la manière dont le sujet s'est représenté son monde infantile et non à l'environnement dans lequel il a grandi tel qu'aurait pu le décrire un observateur indépendant. Freud ne s'est pas intéressé aux troubles graves du développement de l'enfance et, comme tout le monde, de son temps, il aurait tenu probablement nos autistes pour des idiots sinon pour des dégénérés. C'est une psychanalyste de troisième génération, Klein, qui, en 1929, avec son célèbre cas « Dick», a décrit probablement le premier cas d'autisme qu'elle appelle schizophrène [24]. Il est remarquable que, dans la présentation qu'elle fait de ce cas au Congrès international de psychanalyse d'Oxford, elle ne porte aucun jugement négatif sur la mère dont elle comprend la très naturelle angoisse. Elle attribue les particularités de cet enfant, qu'elle a pris en traitement, semble-t-il avec d'assez bons résultats, à une disposition innée, à une incapacité constitutionnelle à tolérer l'angoisse et à la projeter dans les objets qui l'entoure, donc à symboliser l'angoisse dans le jeu avec ces objets, procédé utilisé par les autres enfants pour maîtriser leurs difficultés émotionnelles.

D'autres psychanalystes sont restés tout aussi prudents. Ainsi, Mahler parle, elle aussi, de disposition innée, en allemand Anlage, et affirme qu'elle a rencontré des mères de toutes sortes. Utilisant la technique des thérapies mèreenfant, elle cherche à rétablir un lien entre le bébé et sa mère, mais sans attribuer à la mère une responsabilité unilatérale dans un dysfonctionnement interactif qu'elle affirme plutôt lié à l'incapacité du bébé à réagir aux offres maternelles et à savoir utiliser sa mère comme organisateur de sa vie émotionnelle anarchique. Inspirée à la fois par Mahler et par Klein, Tustin, si elle distingue un autisme psychogène pur des traits autistiques associés à des pathologies organiques reconnues, si elle évoque une possible dépression transitoire de la jeune mère qui la rend moins capable d'accompagner son enfant dans la difficile épreuve du sevrage, insiste, comme son contemporain Meltzer, sur l'intolérance à la frustration native des futurs autistes $[28,38]$. C'est cette intolérance qui les rend incapables de renoncer à la continuité sein-bouche et les plonge dans un vécu d'arrachement et de mutilation au moment de la séparation d'avec le mamelon. C'est encore cette intolérance qui les engage ensuite dans un processus de démantèlement psychique, contre lequel ils essaient de se défendre par un retour autosensuel à la fascination par des formes autistiques, par des stéréotypies ou par la manipulation d'objets autistiques. Au même moment, des auteurs français, dans la suite des travaux de Julian de Ajurriaguerra, ont insisté sur l'intrication complexe, chez le bébé, du développement neurologique et du développement psychique. C'est peut-être René Diatkine qui a le mieux résumé leur position sur l'autisme. Selon lui, le premier développement de l'enfant est guidé par un équilibre entre deux tendances adverses: une tendance à explorer la nouveauté, l'imprévu, une tendance à rétablir l'homéostasie, le connu. L'enfant autiste souffre d'un déséquilibre entre ces deux tendances qui le mène à privilégier l'immuable et que l'environnement échoue à compenser, voire qu'il peut involontairement aggraver, chaque stimulation nouvelle étant vécue par l'enfant sur le mode d'un traumatisme (ce qui justifiait, aux yeux de Diatkine, une guidance précoce des parents et ensuite une aide spécialisée pour aider l'enfant à apprivoiser, sur un mode progressif et non destructeur, la nouveauté).

On est loin, on le voit de la culpabilisation alléguée des familles. Alors d'où vient ce reproche si universellement répété ? Il est de fait que, contrairement aux auteurs qui 
viennent d'être cités, d'autres psychanalystes ont voulu relier directement l'autisme soit à un dysfonctionnement familial global, soit à une pathologie maternelle masquée. Contemporain des premiers travaux de Kanner, un certain nombre de psychanalystes américains, accoutumés à distinguer de manière rigide les troubles qu'ils qualifiaient de «fonctionnels», donc curables, des troubles dits « lésionnels », donc incurables, s'étaient, comme Kanner, attachés à repérer chez les parents des traits de caractère et des comportements susceptibles de barrer l'accès au complexe d'Edipe et de maintenir une fixation ou de déterminer une régression vers des phases archaïques, dites prégénitales, du développement [13]. L'absence d'autorité du père et l'excès de présence d'une mère angoissée étaient donc considérés comme pouvant être générateurs d'autisme ou de psychose. Concrètement, cette position engageait à proposer aux parents des cures individuelles ou des thérapies familiales associées à la prise en charge de l'enfant. Ces propositions, souvent pleines de bonnes intentions, furent, un temps, assez bien acceptées par les familles américaines des classes moyennes, avant de donner naissance, comme on l'a vu, à un mouvement de rébellion. D'emblée, elles suscitèrent en France, où la psychanalyse était alors moins acclimatée, une forte résistance. Le conflit s'aggrava notamment sous l'influence de deux personnalités.

Aux États-Unis, un émigré juif autrichien, Bettelheim, éducateur formé à la psychanalyse plutôt que psychanalyste stricto sensu, avait été chargé de la direction d'une école pour enfants en difficultés émotionnelles en même temps que d'un poste de professeur de sciences de l'éducation à l'université de Chicago. Dans cette école, qui servait de lieu de stages pour ses étudiants et qui fonctionnait principalement sur le mode de l'internat, il avait accueilli quelques enfants autistes auxquels il proposait un environnement structuré pour permettre l'expression de leurs émotions et le rétablissement d'une communication. Très marqué par une expérience concentrationnaire à Dachau et à Buchenwald, auteur d'un livre sur la psychologie du déporté, il avait utilisé une comparaison malheureuse avec les camps pour décrire le monde tel que l'enfant autiste se le représentait : un monde sur lequel on n'a aucune influence, auquel on ne peut que se soumettre en renonçant à tout espoir, ce qu'il appelait une situation extrême. Bien qu'il ait déclaré avec insistance que ce monde n'était pas nécessairement le monde réel dans lequel l'enfant avait vécu, qu'aucun parent n'avait souhaité avoir un enfant autiste et bien qu'il se soit élevé contre la mise en cause unilatérale des mères, son obstination à préconiser un traitement en internat et la séparation d'avec la famille, ainsi qu'un certain nombre de phrases outrancières sur les désirs de mort inconscients de certaines mères, firent de lui le modèle du psychanalyste culpabilisateur [6]. Après sa mort tragique (le suicide d'un vieil homme physiquement très diminué, reclus dans une maison de retraite), une campagne de presse l'accusa d'avoir menti sur ses diplômes, d'avoir truqué ses résultats et fit paradoxalement de lui une sorte de bourreau comparable aux nazis dont il avait été la victime. On en oublia du coup tout son apport au traitement institutionnel des enfants autistes et psychotiques qui, aujourd'hui encore, doit beaucoup à ses recherches [7].

En France, le livre à succès d'une jeune psychanalyste d'origine belge, Mannoni, élève de Françoise Dolto, publié en 1963, L'enfant arriéré et sa mère, fut une des étincelles qui devaient, quelques années plus tard, mettre le feu aux poudres. Ce livre, où il n'est pas encore directement question d'autisme, apporte un regard nouveau sur la déficience mentale en essayant d'en montrer les ressorts défensifs et, par conséquent, améliorables par un traitement psychothérapique. Il insiste malheureusement dans des termes dogmatiques sur l'effet destructeur de la parole maternelle, soit que cette parole scelle définitivement de lourds secrets qui empêche le développement intellectuel de l'enfant, soit qu'elle véhicule une fantasmatique inconsciente qui lui interdit le droit à sa propre parole, l'incarcère dans la jouissance de la mère ou le précipite pieds et poings liés dans l'abîme ouvert par un désir mortifère [27]. Cette vision de la mère sous les traits d'une Médée meurtrière, reprise par l'auteur dans d'autres travaux, s'imposa à de nombreux professionnels, qui la relayèrent dans leurs équipes et la transmirent, sans preuves, d'une manière quasi terroriste aux familles, tenues, de plus, à l'écart des institutions, sous prétexte d'y préserver un espace pour l'enfant, pur de toute contamination maternelle. Ces excès théoriques firent oublier, là aussi, toute l'œuvre institutionnelle de l'auteur et la création du remarquable établissement qu'est encore l'école expérimentale de Bonneuil. Ils firent oublier aussi les regrets tardifs exprimés par Mannoni elle-même, où elle se déclarait mal comprise et reconnaissait l'inefficacité d'attitudes consistant à culpabiliser les parents [34].

Bien qu'elles fussent minoritaires, bien qu'elles aient été critiquées par d'autres psychanalystes, ce sont pourtant ces deux ouvres, celle de Bettelheim, celle de Mannoni, généralement caricaturées et simplifiées, qui représentent aujourd'hui, aux yeux de l'opinion, le discours psychanalytique dans sa totalité. On ne se préoccupe plus, dès lors, de retourner aux sources, et on ne cite même plus dans l'historique bibliographique, contrairement à ce qui se passe dans d'autres domaines de la science, des travaux qui tenaient encore le haut du pavé, il y a moins de 30 ans. Tenus pour nuls ou non advenus, au même titre que l'alchimie ou l'astrologie, les découvertes cliniques accumulées pendant un demi-siècle de psychanalyse sont tombées dans les poubelles de l'histoire, quand elles ne font pas l'objet d'un interdit évoquant les régimes totalitaires. 


\section{Recherches neurobiologiques}

Qu'y a-t-il en face ? D'abord la proclamation maintes fois répétée, dans les médias, d'une masse considérable de découvertes qui sont censées révolutionner le champ de l'autisme et prouver définitivement que l'autisme est un trouble «neurodéveloppemental », « hautement génétique » (des termes aussi mal définis que celui de "lésion métaphysique » employé par certains psychiatres du XIx ${ }^{\mathrm{e}}$ siècle). Une étude attentive de la documentation disponible rend plus humble, même si de nombreuses recherches sont en cours. On peut les classer en trois grandes catégories.

\section{Recherches sur les structures cérébrales}

Utilisant les moyens d'imagerie moderne et notamment l'imagerie par résonance magnétique (IRM), elles sont de deux ordres : structurelles et fonctionnelles. Nous avons déjà parlé de l'hypothèse d'une anomalie de fonctionnement et de structure de la substance réticulée du tronc cérébral. Elle attribuait l'autisme à un trouble du filtrage des sensations du monde extérieur, excès ou, au contraire, déficit de ce filtrage, ou encore désorganisation des sensations avant qu'elles atteignent le cortex. Cette hypothèse n'est plus guère d'actualité. Sur un très petit nombre de cas, un neuroradiologue américain, Courchesne, avait eu son heure de célébrité en mettant en cause la partie médiane du cervelet, le vermis, dont on sait qu'il est richement connecté avec l'ensemble du système nerveux central [12]. Des travaux ultérieurs n'ont pas confirmé cette constatation, mais l'on voit périodiquement reparaître des publications centrées sur l'étude de cellules cérébelleuses particulières, les cellules de Purkinje, qui jouent un rôle dans l'apprentissage des mouvements. Récemment, le journal Le Monde annonçait la découverte par une équipe française de «stigmates» de l'autisme [32]. L'article scientifique cité semble moins affirmatif. En 2000, l'American Academy of Neurology et la Child Neurology Society, dans un avis conjoint, avaient estimé inutile la pratique systématique dans l'autisme de l'IRM. Cet examen coûteux, qui nécessite, de plus, une sédation importante de l'enfant pour l'empêcher de bouger pendant l'examen, ne donnait que peu de résultats ou des résultats difficilement interprétables. En utilisant une méthode plus sophistiquée, les auteurs, qui ont comparé 77 enfants entre 3 et 16 ans, atteints d'autisme non syndromique (c'est-à-dire sans pathologies neurologiques ou génétiques connues associées) à 77 enfants sans aucun trouble psychiatrique ou neurologique, apportent les constatations suivantes : $10 \%$ des IRM des enfants autistes sont ininterprétables, parce qu'elles montrent des anomalies à la limite auxquelles il est impossible d'accorder une signification; dans ce qui reste, $48 \%$ des enfants ont des anomalies et $52 \%$ ont une IRM normale. Les anomalies sont de trois types : une modification de la substance blanche qui pourrait traduire un trouble sous-jacent de la connectivité cérébrale, un élargissement des espaces de Virchow-Robin, des espaces entourant les artères qui pénètrent dans le cerveau, enfin des anomalies diverses siégeant dans le lobe temporal. Aucune de ces anomalies n'est spécifique de l'autisme et peut se retrouver dans de multiples troubles métaboliques ou génétiques ou dans certaines atteintes virales. Les auteurs, conscients de l'aspect disparate de leurs découvertes, insistent surtout sur l'intérêt de pratiquer systématiquement cet examen qui, associé à d'autres constatations cliniques ou biologiques, permettrait peut-être de contribuer à préciser l'étiologie [8].

D'autres examens d'IRM ayant montré un épaississement du sillon temporal supérieur chez un petit nombre d'autistes, on a engagé des études d'IRM fonctionnelle (une méthode qui met en évidence l'activation de certaines zones cérébrales pendant l'exécution de certaines tâches) chez des autistes adultes d'assez bon niveau. Il faut en effet obtenir la coopération du patient dans ces études qui, à la différence de l'IRM anatomique, ne peuvent se faire sous sédation. L'étude consistait à étudier l'activation de la zone à l'audition de la voix humaine ou à celle d'un bruit quelconque. On a remarqué alors que sur six autistes comparés à six sujets normaux volontaires, deux réagissaient comme les normaux, en activant cette zone de manière privilégiée à l'audition de la voix humaine, alors que quatre réagissaient indifféremment à la voix humaine et à un bruit quelconque. En dépit de la modestie de ce résultat (qui a toutefois l'intérêt de pouvoir être rapproché d'autres études sur la perception de la physionomie et la reconnaissance des émotions sur un visage), la presse s'en est emparée pour multiplier des annonces triomphalistes sur la solution de l'énigme de l'autisme [15].

De nombreuses autres hypothèses ont été proposées : un trouble distribué de la connectivité cérébrale, une croissance prématurée et excessive du cerveau. Elles inspirent des travaux, mais aucune ne paraît aujourd'hui s'imposer par rapport aux autres.

\section{Recherches génétiques}

Un certain nombre d'arguments ont fait envisager, depuis longtemps, l'intervention de facteurs génétiques dans l'autisme : la disparité de la distribution entre les deux sexes (environ une fille pour quatre garçons), l'augmentation de la prévalence chez les apparentés multipliée par dix (ce qui, pour un trouble qui, jusqu'il y a peu, restait rare, laissait le risque assez bas), la concordance entre les vrais jumeaux qui ont, comme on sait, le même génome. Mais le fait que le pourcentage chez les apparentés n'obéisse pas aux lois de l'hérédité mendélienne, qui devraient donner une fréquence très supérieure, le fait que chez les vrais 
jumeaux, 10 à $40 \%$, selon les études, de jumeaux d'autistes ne sont pas autistes, montrent que d'autres facteurs que génétiques peuvent intervenir. On parle alors de « susceptibilité », de "vulnérabilité », de "prédisposition », termes assez vagues qui ne sont pas sans rappeler la diathèse des anciens auteurs, qui était surtout une catégorie d'attente dont des découvertes ultérieures devaient souvent faire justice (comme la fameuse diathèse tuberculeuse disparue avec la découverte du bacille de Koch). Ces restrictions à l'enthousiasme du tout génétique, auxquelles il faut ajouter les travaux modernes sur l'épigenèse, qui atténuent l'opposition traditionnelle entre l'inné et l'acquis, sont reconnues par tous les chercheurs sérieux, mais sont passées sous silence tant dans les médias que sur les sites Internet.

Parce qu'on avait trouvé dans les plaquettes sanguines d'un certain nombre d'autistes un taux plus élevé de sérotonine, on s'est un temps intéressé à un gène codant une protéine utilisée dans la synthèse de la sérotonine à partir du tryptophane. Ces recherches ne semblent pas avoir mérité l'annonce faite alors à grand son de trompe, dans la presse, de la découverte du gène de l'autisme.

Plus récemment, une intéressante étude portant sur une famille où un des frères était atteint d'autisme et l'autre d'un syndrome d'Asperger a montré, dans cette famille, les modifications sur le chromosome $\mathrm{X}$ de deux gènes codant pour les neuroligines, des substances engagées dans la formation et dans le fonctionnement des synapses. Les auteurs ont alors réussi à modifier l'un des gènes codant pour une neuroligine chez une souris et remarqué que cette souris recherchait moins le contact d'une autre souris placée dans un compartiment voisin [10]. On a aussi évoqué des mutations ou des délétions de gènes d'autres chromosomes $2,7,15,22$. Avec la multiplication des patients atteints de troubles du spectre autistique et l'estompage progressif des limites de ce spectre, le risque de découvrir une anomalie chez les apparentés s'accroît de jour en jour, rappelant l'époque où il suffisait d'avoir un lointain cousin coureur de filles ou louchant un peu pour être taxé d'appartenance à un rameau dégénéré de l'espèce humaine.

Les stigmates ne sont pas toujours des défauts et le $\mathrm{xIX}^{\mathrm{e}}$ siècle faisait aussi le rapprochement entre les signes de dégénérescence et le génie. On peut trouver un écho contemporain de ce rapprochement dans l'hypothèse d'un psychologue anglais, Baron-Cohen. Comme Asperger jadis, il a cru noter une plus grande proportion d'ingénieurs et de mathématiciens chez les pères d'autistes. Il a alors voulu distinguer deux formes d'intelligence: une intelligence masculine, orientée vers les mathématiques, la mécanique et les problèmes de logique, une intelligence féminine orientée vers l'intuition et la perception des émotions. Ces différences dans le maniement des concepts et des affects correspondraient à des différences observées sur des moyennes d'images cérébrales en IRM. Individuellement, chacun de nous serait un mixte d'intelligence masculine et féminine, mais, dans les généalogies d'autistes, on pourrait repérer un cerveau hypermasculin, ce que confirmeraient certaines recherches relevant une inondation exagérée par la testostérone maternelle des fotus futurs autistes [3].

Retrouvant à un autre niveau l'hypothèse de la responsabilité maternelle, des travaux récents se penchent sur la possibilité d'un mécanisme immunitaire et d'une incompatibilité fotomaternelle. Ils ne paraissent pas faire l'objet d'un scandale. Imaginer que la mère puisse rejeter son foetus avec ses anticorps est probablement moins stigmatisant que d'attribuer un rejet à son inconscient !

\section{Données des recherches en psychologie cognitive}

Ce sont probablement les plus intéressantes, à la fois pour la connaissance et pour la pratique. Dès les années 1980, une équipe anglaise avait mis en évidence, chez un certain nombre d'autistes, ce qu'elle appelait un « défaut de théorie de l'esprit », une difficulté à se représenter et à comprendre les états mentaux d'autrui [2]. Différents tests dits «de fausse croyance » cherchaient à mettre cette difficulté en évidence. Elle semblait signer le dysfonctionnement d'un module cérébral particulier, un réseau hypothétique de neurones formant une sorte d'organe nécessaire à la connaissance d'autrui. Cet organe était supposé se développer progressivement à partir de l'intérêt porté par l'enfant à la direction du regard de l'adulte nourricier puis de la désignation par cet enfant d'un objet extérieur pour attirer le regard d'autrui, ce qu'on appelle l'attention conjointe. Il était à la base du développement du jeu symbolique. La défaillance précoce de ces fonctions est considérée aujourd'hui comme un signe de dépistage précoce d'un risque autistique.

La notion de «module de la théorie de l'esprit» est aujourd'hui discutée, comme du reste toute la théorie modulaire de l'esprit, cette nouvelle phrénologie que l'on devait au philosophe américain Jerry Fodor. Certains, retrouvant la définition de Kanner d'un « trouble inné du contact affectif » et la notion de «balise d'orientation émotionnelle » de Mahler, mettent en cause l'incapacité de l'enfant à reconnaître les émotions sur le visage maternel et à utiliser cette reconnaissance pour orienter son comportement [18]. Ils notent que, lorsqu' on lui demande d'imiter un comportement, l'enfant autiste peut l'imiter grossièrement, mais qu'il a de la difficulté à en imiter le style, comme s'il n'en percevait pas l'intention [19]. Ils remarquent qu'il regarde seulement le comportement du modèle, alors que des enfants non autistes du même niveau échangent des regards avec le modèle comme s'ils cherchaient à deviner son but [39]. La découverte des systèmes miroirs et les travaux neurophysiologiques sur l'empathie, dont il a déjà été question, amènent à replacer la question dans le cadre 
plus général d'une déficience des réseaux distribués à la base de l'intersubjectivité.

D'autres travaux portent sur les modalités perceptives particulières des autistes. Ils ont montré la difficulté de l'autiste à apprécier globalement une figure et, au contraire, ses capacités supérieures à celles de sujets non autistes dans la perception des petits détails. C'est ce qui explique, chez certains d'entre eux, des talents paradoxaux comme l'oreille absolue en musique, ou la mémoire exceptionnelle de dates ou de listes d'annuaires. On a pu parler à leur propos d'une « autre intelligence» [31]. On a pu rapprocher aussi leur désir d'immuabilité d'une meilleure perception des formes fixes et d'une «malvoyance » du mouvement [17].

Ces travaux, dont on ne peut donner ici qu'un trop rapide aperçu, ne s'opposent pas aux conceptions psychanalytiques et semblent apporter des bases plus rigoureuses aux intuitions des cliniciens. Curieusement, ils font beaucoup moins l'objet de publicité que des recherches génétiques ou neuroradiologiques dont les résultats sont pourtant plus fragiles et ont moins de conséquences pratiques.

\section{Prises en charge}

C'est encore un sujet de controverses et, très naturellement, celui où les associations de parents se sont engagées avec le plus de passion.

\section{Prises en charge relationnelles d'inspiration psychodynamique}

Une clarification s'impose. Il convient de distinguer la psychanalyse en tant que cure de l'utilisation de la théorie et des modalités d'écoute psychanalytiques dans les prises en charge institutionnelles.

Contrairement à ce qui est souvent affirmé, il y a eu peu de psychanalystes qui se soient engagés directement, comme l'avaient fait Klein, Mahler, Tustin et le groupe réuni autour de Meltzer, relayés en France, par Haag et Houzel, dans la cure psychanalytique authentique, à plusieurs séances par semaine, d'enfants autistes [22]. D'une part, cet engagement nécessite de la part des analystes des qualités particulières. D'autre part, il y a encore probablement peu d'enfants disposant des capacités intellectuelles et verbales nécessaires pour suivre un tel traitement et peu de familles disposées à en accepter la charge. Il est, par ailleurs, légitime de se demander, mais ce serait l'objet d'une longue discussion, si le qualificatif de psychanalytique est bien adapté à ces cures. Ne s'agit-il pas plutôt de psychothérapies intensives et prolongées, s'appuyant sur la métapsychologie freudienne, mais utilisant aussi des mouvements contre-transférentiels spécifiques, un échange d' «affects de vitalité » [37] et de plaisir à jouer et à comprendre, une créativité particulière et la mise en œuvre, chez l'analyste, de procédés narratifs (le récit fait à l'enfant de ce qui se passe entre le thérapeute et lui) plutôt que d'interprétations des motions inconscientes ? Ces traitements analytiques, bien qu'étant restés en nombre limité, nous ont beaucoup appris et continuent à nous apprendre beaucoup sur le fonctionnement mental des autistes, dont la connaissance est aussi enrichie par les témoignages écrits des autistes capables de décrire leur expérience.

Grâce à toutes ces données, des approches relationnelles, multidimensionnelles et intégrées ont pu se multiplier. Elles associent des thérapies individuelles et de groupe, des ateliers d'expression, des rééducations orthophoniques ou psychomotriciennes, à une éducation et à une pédagogie adaptée, au sein d'institutions de proximité de plus en plus souples et diversifiées, en fonction d'un projet individualisé $[20,29]$. Nous avons déjà mentionné les hôpitaux de jour et les institutions médicoéducatives. Différents types de soins à temps partiel sont venus s'y adjoindre, ainsi que des prises en charge ambulatoires en lien avec l'insertion dans des milieux sociaux normaux : l'école, les centres de loisir, culturels et sportifs. L'intégration scolaire des enfants souffrant d'autisme ou de troubles associés, codifiée par des circulaires conjointes des ministres de la Santé et de l'Éducation nationale du début des années 1980 et entreprise à l'initiative des équipes psychiatriques ou psychopédagogiques, a ainsi fait l'objet de plusieurs expériences de pointe évaluées dans un réseau de recherche Inserm [9]. La France, mal défendue par des pouvoirs publics, apparemment peu soucieux de promouvoir et de valoriser le travail de leurs agents, peut, contrairement aux condamnations prononcées par des organismes visiblement informés de manière unilatérale, aborder sans honte, de ce point de vue, la comparaison avec d'autres pays, même si, comme ailleurs, la crise économique et les restrictions budgétaires freinent un élan militant qui s'était engagé après la Libération, même si persiste encore, il faut l'avouer, dans quelques poches, la dérive sectaire qui a perverti l'inspiration psychanalytique, même si surtout l'équipement relativement correct en matière de petite enfance a laissé hors de sa visée thérapeutique et éducative un grand nombre d'autistes adolescents et un plus grand nombre d'adultes, notamment les plus dépendants et les plus en attente de soins.

Ce qui fait le fond de l'inspiration psychanalytique et relationnelle, c'est d'abord, pour les professionnels, un travail constant sur eux-mêmes, dans des réunions d'élaboration de leur pratique, où ils s'efforcent de développer, avec leur créativité, leurs capacités d'accueil et d'empathie, en dépistant en eux et en corrigeant les inévitables contreattitudes de fascination ou, au contraire, de rejet suscitées par une rencontre ouverte avec l'enfant et sa famille. C'est 
ensuite le souci de comprendre, par-delà les symptômes, la valeur qu'ils ont pour l'enfant, de les envisager non seulement comme l'expression d'un déficit, ce qu'ils sont nécessairement, mais aussi comme le produit d'un processus organisé pour compenser spontanément ce déficit, ce que Mahler appelait des «mécanismes de survie », bref pour se «défendre » contre les effets du déficit, et notamment contre les angoisses liées à ce déficit et à la confrontation à un monde que ce déficit rend difficilement maîtrisable. Ce qui caractérise encore cette inspiration c'est la prise en compte de tous les comportements de l'enfant, verbaux et non verbaux, dans sa famille et au dehors, dans la séance de thérapie, dans le groupe d'activités, dans la classe ou en promenade, avec le souci de repérer, derrière les anomalies de ces comportements, l'indice d'une communication maladroite de la vie intérieure. C'est aussi, pour dominer le morcellement autistique et l'absorption de l'enfant dans l'instant, l'organisation du vécu intersubjectif dans un récit, sans cesse raconté et reraconté à l'enfant et à ses parents, où les événements de la vie quotidienne, articulés entre eux, prennent sens les uns par rapport aux autres. Il est à remarquer que les conceptions modernes du handicap ainsi que les données les plus récentes de la neurophysiologie du cerveau, qui amènent à distinguer les circuits de l'agentivité automatique immédiate de ceux qui produisent la conscience temporelle de l'auteur de l'acte, engagé dans son histoire personnelle, sont cohérentes avec cette optique. Elle s'oppose à celle qui tend à prévaloir aujourd'hui, en particulier sous la pression des associations de familles (ou de certaines d'entre elles).

\section{Prises en charge éducatives}

Elles reposent sur deux postulats.

Le premier correspond à ce qui s'est appelé l' « idéologie de la normalisation ». Il limite le handicap à une seule de ses composantes, le désavantage social qu'il entraîne. Il affirme que l'adaptation du milieu aux handicapés et le refus de la stigmatisation opérée par les institutions spécialisées suffisent à faire disparaître le handicap. De même qu'un invalide en fauteuil roulant ne souffre plus d'une déclivité dès lors qu'un plan incliné lui permet de la franchir plus aisément que s'il devait gravir des escaliers, de même, croit-on, l'insertion scolaire tous azimuts des enfants autistes, avec la seule assistance d'auxiliaires à la vie scolaire très peu formés, suffit à leur permettre de suivre le cursus des autres enfants. Cette idéologie de la normalisation, très en vogue au Canada et en Italie, offre d'incontestables avantages économiques en diminuant le recours onéreux à des professionnels de haut niveau. Elle donne aussi aux parents l'illusion d'avoir trouvé pour leur enfant une solution qui se révèle bien vite être un leurre. Elle est, à l'heure actuelle, de plus en plus appréciée par la hiérarchie de l'Éducation nationale, qui retrouve le pouvoir sans partage sur l'enfance à laquelle elle aspire depuis Jules Ferry et qu'elle a toujours souffert de se voir disputé par les médecins et les autres thérapeutes. Il suffit de se souvenir du combat sans merci livré, au début du $\mathrm{xx}^{\mathrm{e}}$ siècle, par l'inspirateur des classes de perfectionnement, Alfred Binet, contre Désiré Magloire Bourneville, le médecin créateur de l'approche médicopédagogique destinée à ceux qu'on appelait alors les idiots et les imbéciles. Après avoir transformé l'asile de Bicêtre en une institution dynamique pour ces enfants, Bourneville avait commencé à créer, pour les plus évolutifs d'entre eux, des classes spéciales annexées à des écoles de Paris où les enseignants collaboraient avec les médecins. Il fut contré avec succès par Binet, qui préférait renvoyer les enfants lourdement perturbés dans des garderies restreintes en personnel et réserver l'école aux enfants apparemment plus doués que, sur la foi de son échelle de mesure de l'intelligence, il estimait perfectibles. En même temps que les enfants les plus atteints, il excluait de l'école les médecins qui s'en occupaient. C'est une tendance analogue (même si elle est apparemment plus inclusive) qui se dessine aujourd'hui, laissant les enseignants de base sans soutien intellectuel et sans aide psychologique (et souvent sans outils pédagogiques adéquats) face à des enfants dont les stratégies d'apprentissage très particulières leur sont souvent impénétrables. Elle risque de conduire à une nouvelle ségrégation.

Cependant, comme la réalité du handicap ne peut être éliminée par un simple tour de passe-passe, on prétend qu'il suffit de la minimiser en la contrôlant. Les seules méthodes admises sont alors des méthodes d'apprentissage inspirées d'une théorie scientifique obsolète : le comportementalisme. Élaborée au début $\mathrm{du} \mathrm{xx}^{\mathrm{e}}$ siècle par un psychologue expérimentaliste, John Broadus Watson, elle assimilait le fonctionnement mental au fonctionnement réflexe en n'étudiant que le stimulus et la réponse. L'évolution des neurosciences, grâce en particulier aux moyens modernes d'investigation fonctionnelle, a conduit à abandonner ce paradigme et à faire une place importante à l'activité spontanée du cerveau. Cette théorie a néanmoins continué à inspirer des pratiques en utilisant notamment le conditionnement opérant, c'est-à-dire le renforcement d'une action souhaitée par une récompense et sa disparition par des sanctions (directes ou indirectes). Ces pratiques ne sont finalement qu'une codification plus ou moins sophistiquée des procédés qui forment, de toute éternité, la base du dressage et de cet élément de dressage qui figure dans toute éducation. Systématisées, dans les années 1960, sous le nom d'ABA (applied behavioral analysis) pour contrôler une gamme de comportements non souhaitables : la délinquance, l'addiction, l'alcoolisme mais aussi, à l'époque, l'homosexualité, elles ont été appliquées à l'autisme par un psychologue américain Lovaas. Longtemps très discutées, elles redeviennent à la mode après une longue période de 
latence, sous la forme d'exercices intensifs, centrés sur des symptômes ciblés et couvrant, dans leur forme la plus complète, 40 heures par semaine de face-à-face de l'enfant autiste avec un parent ou une série d'auxiliaires qui se succèdent (ce qui évite un attachement individualisé de l'enfant à un thérapeute, contrairement aux approches psychodynamiques à l'origine, chez certains parents, d'un sentiment de concurrence) [25]. L'ABA se présente comme la seule méthode validée scientifiquement, mais lorsqu'on se penche sur les articles écrits par ses promoteurs, on s'aperçoit que l'efficacité validée porte sur quelques comportements comme l'écholalie, l'automutilation, les crises d'agitation ou le repli autosensuel. On peut aussi se demander si le simple fait d'accorder à l'enfant autant d'attention et de partager avec lui de nombreux échanges, dans une atmosphère de plaisir commun (plaisir de l'enfant à être récompensé, plaisir de l'adulte à voir l'enfant performer dans le sens souhaité, réverbération en miroir de ces deux types de plaisir) ne suffit pas à expliquer les bons résultats.

La méthode ABA supplante aujourd'hui une autre méthode naguère très prônée : le programme TEACCH. Il s'agit en fait plus d'un programme de santé publique proposé en 1970 par Schopler à l'État de Caroline du Nord et importé, en France, dans les années 1980 par des parents qui se rebellaient contre l'omniprésence de la psychanalyse dans les institutions françaises [36]. Ce programme, qui prévoyait d'articuler des centres de dépistage, des écoles spécialisées, des centres pour adolescents et pour adultes et d'accompagner, tout au long de la vie, la socialisation des autistes, reposait plus sur une série de principes que sur des méthodes spécifiques. Le premier principe était d'associer très étroitement aux prises en charge techniques des parents que les institutions d'inspiration psychanalytique avaient alors trop tendance à tenir à distance. Le deuxième principe consistait à pratiquer une évaluation très précise des déficits de l'enfant et surtout de ses émergences dans des domaines où, bien qu'encore en échec, il témoignait d'un début d'ouverture. Le troisième principe consistait à structurer l'environnement et à individualiser les tâches prescrites à l'enfant en tenant compte de ses émergences mais en évitant de le noyer sous un flux de stimulations (en particulier verbales). Présentés malheureusement sur un mode polémique, ces principes de bon sens ont tardé à pénétrer dans les institutions françaises. Aujourd'hui, ils connaissent un regain d'intérêt et, dans une atmosphère dépassionnée, inspirent de plus en plus une approche éducative qui se conjugue à l'approche thérapeutique [11].

\section{Conclusion}

C'est sur cette amorce de consensus que nous voudrions conclure. L'autisme, en quelque sorte, est contagieux et a toujours eu tendance à susciter des exclusives qui conduisent chacun des protagonistes à s'enfermer dans l'ignorance du point de vue d'autrui, avec la certitude d'être seul à posséder la vérité. On commence à comprendre que de tels clivages, très préjudiciables tant à une politique cohérente de santé et d'éducation qu'à la recherche, dans un domaine encore très obscur, ne sauraient longtemps persister. L'heure est peut-être venue de renoncer à se disputer sur une origine dont on ne sait pas grand-chose et de reconnaitre modestement dans l'autisme, en attendant mieux, une « voie finale commune », un mode de réaction de l'enfant à une multitude de facteurs innés ou acquis, structurels ou fonctionnels, génétiques ou environnementaux, probablement intriqués. L'heure est peut-être venue de se demander s'il n'y a pas un mais, peut-être, plusieurs autismes, et s'il ne faudra pas un jour, prenant le risque impopulaire de diminuer la prévalence du «spectre autistique », établir des sous-catégories sans grand rapport les unes avec les autres. L'heure est surtout venue de mettre un terme à une bataille où se dépense une énergie qui serait mieux employée à promouvoir des pratiques de réseau articulant le soin avec l'éducation et la pédagogie.

\section{Références}

1. Asperger H (1944) Les Psychopathies autistiques pendant l'enfance, tr. fr. (1998). Le Plessis Robinson. Les Empêcheurs de penser en rond, Synthélabo

2. Baron-Cohen S, Leslie A, Frith U (1985) Does the autistic child have a theory of mind? Cognition 21:37-46

3. Baron-Cohen S (2002) The extreme male theory of autism. Trends Cogn Sci 6:248-54

4. Bender L (1947) Childhood schizophrenia. Clinical studies of one hundred schizophrenic children. Am J Psychiatry 17:40-56

5. Berquez G (1983) L'autisme infantile. Introduction à une clinique relationnelle selon Kanner. PUF, Paris

6. Bettelheim B (1967) La forteresse vide, tr. fr. (1969). Gallimard, Paris

7. Bettelheim B (1950) L'amour ne suffit pas. Le traitement des troubles affectifs de l'enfant, tr. fr. (1970). Fleurus, Paris

8. Boddaert N, Zilbovicius M, Philipe A, et al (2009) MRI findings in 77 children with non-syndromic autistic disorder. PLoS ONE 4(2):e4415. doi:10.1371/journal.pone.0004415

9. Botbol M, Tordjman S, Lauth B, Ferrari P (1994) L'autisme et les psychoses précoces. Réseau Inserm. La recherche en psychiatrie, expériences, méthodes, perspectives. Rev Inserm

10. Bourgeron T, Leboyer M, Delorme R (2009) Autisme, la piste génétique. Recherche 426:41-3

11. Constant J (1997) La méthode TEACCH dans un hôpital de jour public. À propos d'une expérience concrète. In: Misès R., Grand P. (eds) Parents et professionnels devant l'autisme. Éditions du CTNERHI, Paris

12. Courchesne E, Yeung-Courchesne R, Press GA, et al (1988) Hypoplasia of cerebellar vermal lobules VI and VII in autism. N Eng J Med 318:1349-54

13. Despert JL (1951) Some considerations relating to the genesis of autistic behavior in children. Am J Orthopsychiatry 21:335-50

14. Freud S (1896) L'hérédité et l'étiologie des névroses. Rev Neurol 4(6):161-9 
15. Gervais H, Belin P, Boddaert N, et al (2004) Abnormal cortical voice processing in autism. Nat Neurosci 7(8):801-2

16. Georgieff N, Jeannerod M (1998) Beyond consciousness of external reality. A "who" system for consciousness of action and self-consciousness. Conscious Cogn 7:465-77

17. Gepner B (2005) La malvoyance du mouvement dans l'autisme. De la clinique à la recherche et à la rééducation. In: Berthoz $\mathrm{A}$, Barthelemy $\mathrm{C}$, Rogé $\mathrm{B}$, et al. L'autisme. De la recherche à la pratique. O. Jacob, Paris

18. Hobson P (1993) The autistic child appraisal of expressions of emotions. J Child Psychol Psychiatry 27:321-42

19. Hobson P, Hobson JA (2008) Dissociable aspects of imitation: a study in autism. J. Exp Psychol 101:170-85

20. Hochmann J (1997) Pour soigner l'enfant autiste. O. Jacob, Paris

21. Hochmann J (2009) Histoire de l'autisme. O. Jacob, Paris

22. Houzel D, Haag G (1990) La place de la psychanalyse dans l'approche de l'autisme infantile. In: Parquet PJ, Bursztejn C, Golse B (eds) Soigner, éduquer l'enfant autiste ? Masson, Paris

23. Kanner L (1943) Autistic disturbances of affective contact. Nerv Child 2(3):217-50

24. Klein M (1929) The importance of symbol formation in the development of the ego. In: Klein M. (ed) Essais de psychanalyse, tr. fr. (1968). Payot, Paris

25. Leaf R, Mac Eachin J (2006) Autisme et ABA. Une pédagogie de progrès, tr. fr. Pearson Éducation, Paris

26. Mahler M (1952) On child psychosis and schizophrenia - autistic and symbiotic infantile psychosis. Psychoanal Study Child 7:266305
27. Mannoni M (1964) L'enfant arriéré et sa mère. Le Seuil, Paris

28. Meltzer D, Bremer J, Hoxter S, et al (1975) Explorations dans le monde de l'autisme, tr. fr. (2004). Payot, Paris

29. Misès R, Bailly-Salin MJ, Bréon S, et al (1980) La cure en institution. L'enfant, l'équipe et la famille. ESF, Paris

30. Misès R, Quemada N (1993) Présentation de la classification. In: Classification française des troubles mentaux de l'enfant et de l'adolescent. Troisième édition. Ministère des Affaires sociales, Paris

31. Mottron L (2004) L'autisme : une autre intelligence. Mardaga, Sprimont (Belgique)

32. Nau JY. L'imagerie cérébrale dévoile les stigmates de l'autisme. Le Monde numéro du 13 février 2009

33. Rimland B (1963) Infantile autism. Methuen, Londres

34. Rizzo L (2007) Entretien avec Maud Mannoni 1982. Figures de la psychanalyse 14:135-50

35. Rizzolati G, Fadiga L, Gallese V, Fogassi L (1996) Premotor cortex and the recognition of motor action. Cogn Brain Res 3:131-41

36. Schopler E, Reichler J, Lansing M (1991) Stratégies éducatives de l'autisme, tr. fr. Masson, Paris

37. Stern D (1985) Le monde interpersonnel du nourrisson, tr. fr. (1989). PUF, Paris

38. Tustin F (1972) Autisme et psychose de l'enfant, tr. fr. (1977). Le Seuil, Paris

39. Vivanti G, Aparna N, Ozonoff S, Rogers S (2008) What do children with autism attend to during imitation tasks? J. Exp Psychol 101:180-205 